\title{
Die Epidemiologie im Guinness-Buch der Rekorde
}

M it der ISAAC-Studie (International Study of Asthma and Allergies in Childhood) hat es die Epidemiologie allergischer Erkrankungen tatsächlich in das Guinness-Buch der Rekorde geschafft. Sie wurde dort als größte epidemiologische Studie der Welt aufgenommen. Bis heute wurden im Rahmen dieser Studie über 2 Millionen Kinder untersucht.

Ebenfalls mit Ergebnissen der ISAAC-Studie melden wir uns in diesem Heft ab Seite 124 mit Beiträgen der epidemiologischen Jahrestagung vom vergangenen Herbst zurück. Wie wichtig es ist, vergleichbare weltweit erhobene Zahlen zur Häufigkeit allergischer Erkrankungen zu haben, zeigt der Beitrag von Gudrun Weinmayr am Beispiel des Asthmas sehr schön. Zwischen den Ländern bestehen zum Teil erhebliche Prävalenzunterschiede für das Asthma insgesamt und auch für das mit allergischer Sensibilisierung verbundene Asthma. Daran schließt sich die Frage an, welche genetischen und Umwelteinflüsse zu diesen Häufigkeitsunterschieden beitragen.

Thomas Behrens hat in diesem Zusammenhang ebenfalls Daten der ISAACStudie ausgewertet und konnte zeigen, dass die Schimmelpilzbildung in Innenräumen ein wesentlicher Provokationsfaktor für Asthmasymptome darstellt und dass diese Assoziation bei genetischer Vorbelastung noch einmal besonders stark zutage tritt.

Hochinteressante Ergebnisse zur elterlichen Vorbelastung stellt Hermann Pohlabeln am Beispiel der Effekte des Stillens dar. Offensichtlich ist das Stillen bei mütterlicher Vorbelastung mit einem erhöhten und bei väterlicher Vorbelastung mit einem deutlich reduzierten Risiko für Asthma und atopisches Ekzem verbunden. Dies deckt sich mit Befun-

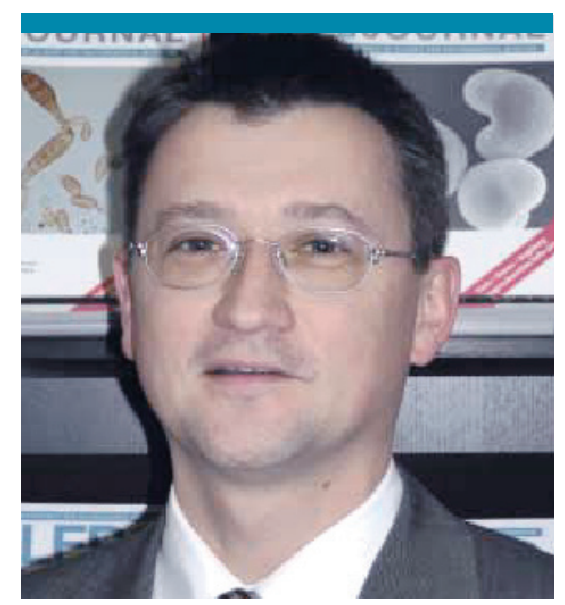

Prof. Dr. Torsten Schäfer, Institut für Sozialmedizin, Universitätsklinikum Schleswig-Holstein, Lübeck, Mitglied im Herausgeberkollegium von Allergo Journal

den, dass eine mütterliche Vorbelastung das kindliche Allergierisiko stärker erhöht als eine väterliche, wobei die zugrundeliegenden Mechanismen noch unklar sind.

Zur Ernährung hat Iris Kompauer einen weiteren sehr interessanten Beitrag vorgelegt, indem sie zeigen konnte, dass die Aufnahme von Antioxidanzien wie Carotinoiden und Tocopherolen negativ mit allergischer Rhinitis und Sensibilisierung verknüpft ist.

In unserer eigenen Kinder von Lübeck Allergieund Umweltstudie (KLAUS) konnten wir am Beispiel der Katze klare Zusammenhänge zwischen Haustierhaltung, Allergenbelastung, allergischer Sensibilisierung und dem Auftreten einer atopischen Erkrankung nachweisen.

Die Bandbreite der epidemiologischen Beiträge reicht bis zur Multiple Chemical Sensitivity (MCS). Eberhard Schwarz hat die Erkrankung bezüglich auslösender Faktoren sehr sorgfältig aufgearbeitet. Schließlich kommen in dem
Beitrag von Angelina Bockelbrink Alternativverfahren und die damit verbundenen Kosten zur Sprache.

Die Epidemiologie hat sich in Deutschland im letzten Jahr als eigenständige Fachgesellschaft etabliert. Während wir bislang von Beiträgen der durch drei Mutterfachgesellschaften getragenen Deutschen Arbeitsgemeinschaft Epidemiologie (DAE) berichteten, wird im September in Greifswald der erste Kongress der Deutschen Gesellschaft für Epidemiologie (DGEpi) stattfinden. Auch die dort vorgestellten allergierelevanten Beiträge werden wir für sie wieder referieren. Sie sind aber auch herzlich zur Tagung eingeladen, um sich einen Überblick über andere interessante Themen wie Ernährung, Krebs, Herz-Kreislauf-Erkrankungen, Infektionen oder Versorgungsforschung zu verschaffen (www.dgepi2006.de).

Abschließend noch eine Bitte: Das Allergo Journal hat eine erfreulich große und stabile Leserschaft. Gerne würden wir mehr darüber erfahren, was Ihnen an unserer Fachzeitschrift gut und was Ihnen weniger gut gefällt. Schreiben Sie uns auch Ihre Anregungen oder Kommentare zu einzelnen Beiträgen. Gerne werden wir Ihre Zuschriften, die von allgemeinem Interesse sind, auch abdrucken.

Jetzt wünsche ich Ihnen viel Spaß bei der Lektüre und bin mit herzlichen Grüßen Ihr

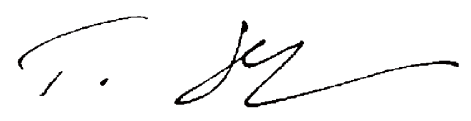

Prof. Dr. Torsten Schäfer, MPH 